Vol. 72, N. ${ }^{\circ}$ II5 (mayo 2020), 7I-79

\title{
ANÁLISIS DE LA ESPECIALIZACIÓN PRODUCTIVA REGIONAL PARA IMPULSAR LA DIVERSIFICACIÓN EXPORTABLE EN ECUADOR
}

\author{
ANALYSIS OF REGIONAL PRODUCTIVE SPECIALIZATION TO BOOST THE \\ EXPORTABLE DIVERSIFICATION IN ECUADOR
}

\author{
BRYAN ESPINOZA ESTRELLA, ${ }^{1}$ JHON PÉREZ ESPAÑA, ${ }^{2}$ JEAN DELGADO LÓPEZ $^{2}$ \\ ${ }^{1}$ Universidad Central del Ecuador, ${ }^{2}$ Investigador independiente
}

Recepción manuscrito: 9 de febrero de 2020

Aceptación versión final: 8 de mayo de 2020

\begin{abstract}
RESUMEN Este trabajo analiza la especialización productiva ecuatoriana frente a Suramérica, a fin de sugerir una mejor diversificación exportable. Para esto, se calculan dos indicadores; por un lado está el índice de sofisticación por producto (PRODY), que indica en qué productos se encuentra especializada Suramérica; y, por otro, está el índice de sofisticación de las exportaciones (EXPY), que señala la especialización por cada país a partir de esa lista de productos regionales. Con respecto a Ecuador, estos índices evidenciaron un bajo nivel de sofisticación de sus exportaciones, lo que conlleva a un limitado crecimiento con respecto a los demás países de la región.
\end{abstract}

PALABRAS CLAVE PRODY, EXPY, sofisticación tecnológica, oferta exportable, especialización productiva.

ABSTRACT This paper analyzes the Ecuadorian productive specialization against South America, in order to suggest a better exportable diversification. For this, two indicators are calculated; On the one hand, there is the product sophistication index (PRODY), which indicates which products South America is specialized in; and on the other, the export sophistication index (EXPY), which indicates the specialization for each country based on that list of regional products. With respect to Ecuador, these indices showed a low level of sophistication in their exports, which leads to a limited growth with respect to the other countries in the region.

KEYWORDS PRODY, EXPY, technological sophistication, exportable supply, productive specialization.

JEL CODES F14, L6, L7.

\section{INTRODUCCIÓN}

La especialización productiva está definida por los bienes y servicios producidos y concentrados alrededor de las capacidades productivas que posee un país o una región; sobre esa 
especialización se definen los ingresos que pueden percibir estas economías. Sin embargo, alcanzar un desarrollo productivo no es fácil, e incluso se vuelve un tema complejo de análisis según la visión teórica por la que se opte. En todo caso, un país se considera con baja sofisticación tecnológica si sus exportaciones están centradas en pocos productos, los cuales a su vez son también producidos por muchas otras economías. Bajo sumatoria, si relacionamos el nivel de ingreso promedio de cada país, asociado al producto, a nivel regional, entonces se obtendría el indicador denominado PRODY «índice de sofisticación por producto».

En otras palabras, el índice PRODY mide el nivel de especialización o de productividad de los bienes, dentro de un contexto regional específico, pero en términos económicos, al encontrarse atado al producto inerno bruto (PIB) per cápita en ese conjunto de países. Por otro lado, si a este índice PRODY, por cada bien respectivo, se lo multiplica al peso ponderado de las exportaciones que realiza uno u otro país, se tendría complementariamente el índice EXPY. Es decir, el EXPY indica el nivel de sofisticación de las exportaciones de un país específico, en el contexto regional, y se lo determina a través de la suma de la renta vinculada a cada producto que ha exportado dicho país. Bajo este antecedente, se torna importante analizar la especialización productiva que tiene la región suramericana a través del índice PRODY; y, sobre ello, a través del índice EXPY, es también importante analizar el nivel de sofisticación de las exportaciones de Ecuador con respecto al resto de países suramericanos.

En general, con este trabajo se evidencia el bajo nivel de sofisticación de la oferta exportable ecuatoriana, y, consecuentemente, se señala el lento crecimiento regional que lleva el país. Al final de esta investigación, se recomienda que Ecuador direccione mejor sus recursos productivos hacia bienes que generen mayor renta per cápita; específicamente, se hablan de los productos que ya estamos produciendo «preparaciones de carne y productos del mar, cacao, grasas y aceites vegetales y animales, productos del sector minero y alimentos preparados para animales», pero es necesario que se incorpore valor agregado y componente tecnológico en sus procesos productivos. Se recomienda también fortalecer la producción minera en una segunda fase. Por último, debe resaltarse que los países que se especializan en productos de alta productividad «con relación a su renta per cápita», desde luego, generarán un mayor impulso en su crecimiento, y con ello, transitarán hacia mejores niveles y condiciones de vida.

\section{MARCO TEÓRICO}

A través del enfoque schumpeteriano se considera que la innovación y la base tecnológica de las empresas determinan el entramado productivo y, a la vez, definen la especialización en un país. Desde el enfoque teórico centro-periferia, en términos de especialización, se indica que los países periféricos no pueden alcanzar un desarrollo productivo debido a la relación desigual con respecto al centro hegemónico industrializado mundial; para ello, los países en vías de desarrollo deberán buscar mayores tasas de productividad e industrialización; y así evitar las pérdidas irremisibles de las ventajas clásicas del intercambio (Prebisch, 1949). A partir de estos primeros argumentos, se puede concluir que los países deben alterar su estructura productiva y encaminarse hacia una especialización real y alcanzable en el corto y mediano plazo. 
Los enfoques económicos fundamentales convergen en aceptar al (PIB) como un indicador indispensable para medir fenómenos pertinentes a la ciencia económica; a su vez, otro indicador es el PIB per cápita; con lo cual ambos son acuñados en general para demostrar la conceptualización teórica del crecimiento económico. Dicho crecimiento, en general, es visto como el cambio porcentual del PIB real de una economía en periodos de tiempo; en el largo plazo se lo logra a través de un crecimiento prolongado. Por esta razón, las instituciones gubernamentales tienden a analizar estas variables como medidas de crecimiento $y$, de ello, tener presente la mejora en el nivel de vida de la población. A nivel mundial, los sistemas productivos son uno de los componentes principales en este andamiaje, con lo cual, son continuamente reestructurados con avances científicos y tecnológicos.

El comercio internacional enfoca a esta reestructuración con dos visiones. En la primera, se considera que la especialización productiva y la necesidad de comercio de los países están dadas por las diferencias en su dotación de factores productivos. En la segunda, propuesta por Paul Krugman, se sugiere que la eficiencia del comercio internacional está dada no solo por la dotación de los factores productivos de los países sino por las economías de escala; pues, este tipo de producción permite la reducción de los costos, y como así también lo afirman Mayorga y Martínez (2008). Con esto, Krugman trata de explicar acerca de la comercialización de aquellas mercancías que se convierten en sustitutos de la producción de una nación con otra; sin embargo, para favorecer estas economías de escala es necesario incorporar mecanismos productivos, tecnológicos y administrativos.

Para Flores y Vaillant (2011, pág. 8), desde la teoría del intercambio comercial entre países, una economía de bajo nivel de sofisticación es aquella que está especializada en la producción de pocos productos, los cuales a su vez son también producidos por muchas otras economías. Para Hausmann, Hwang y Rodrik (2005), la sofisticación de las exportaciones de un país es congruente con aspectos como el contenido tecnológico que tiene un producto, su diseño y la reputación de la marca. Para demostrar estos escenarios, los autores desarrollaron en primer lugar un indicador denominado índice de sofisticación por producto o PRODY, y sirve para calcular la productividad de los bienes de un agregado de países en relación a su PIB per cápita; es decir, el PRODY se lo interpreta como el nivel de ingreso promedio asociado al producto y a nivel regional. Este indicador es mostrado a continuación ${ }^{1}$ donde $x_{k j}$ son las exportaciones del producto k que realiza el país j; $X_{j}$ son las exportaciones totales del país j; y, finalmente, $y_{j}$ es el PIB per cápita del país j.

$$
\operatorname{PRODY}_{k}=\sum_{J} \frac{\left[{ }^{x_{k J}} / x_{J}\right]}{\sum_{J}\left[{ }^{x_{k J}} / x_{J}\right]} y_{J}
$$

En segundo lugar, los autores desarrollaron un indicador que es capaz de aproximar el nivel de productividad de las exportaciones a la renta per cápita dentro de los países, y lo denominaron EXPY o productividad de las exportaciones. Así, el EXPY se determina a través de la suma de la renta vinculada a cada producto exportado, ponderada por la participación del producto en las exportaciones totales del país. Es decir, el ExPY se lo interpreta como el nivel de ingreso 
promedio que tienen los países que exportan una canasta de bienes igual o similar al país que se está analizando. Sobre este indicador, se enuncia que aquellos países que se especialicen en la producción de bienes, con un EXPY más elevado que aquel esperado según su PIB per cápita, estarán en la capacidad de generar un mayor crecimiento económico dentro de la región. A continuación, se presenta este indicador; ${ }^{2}$ donde $x_{k j}$ son las exportaciones del producto k que realiza el país j; $X_{j}$ son las exportaciones totales del país j; PRODY $_{\mathrm{k}}$ es el indicador de sofisticación por cada producto $(\mathrm{k})$, planteado anteriormente.

$$
E X P Y_{J}=\sum_{k}\left(\frac{x_{k_{J}}}{X_{J}}\right) P R O D Y_{k} \text { (2) }
$$

\section{ESPECIALIZACIÓN PRODUCTIVA REGIONAL}

El Observatorio de Complejidad Económica (OCE) ubica a Argentina como la 46. economía de mayor exportación en el mundo (OCE, 2016), con lo cual sus exportaciones para el año 2016 bordearon los USD 129 mil millones; cabe indicar que sus productos provenientes del sector manufacturero industrial y agropecuario participan en el 6o\% dentro de esta oferta exportable. No obstante, entre los años 2006 y 2016, el grado de apertura comercial de la economía argentina alcanzó un promedio de $29 \%$, lo que evidencia una política más centrada en su mercado interno (BCRA, en línea). Brasil, por otro lado, se vincula al comercio exterior a través de exportaciones que provienen del sector agrícola y minero; entre estos productos se destacan la soja, mineral de hierro, petróleo, café y maíz. A pesar de esto, el sector industrial posee un importante valor agregado, lo que le permite también realizar exportaciones de autos, buques, aviones y helicópteros. Es importante resaltar que la producción de Brasil abarca el 60\% del producto total de Suramérica, lo que le guarda un puesto en el grupo de economías emergentes llamado BRICs.

A su vez, Chile, entre los años 2006 y 2016, alcanzó un grado de apertura comercial en torno al 50\%, en gran parte, debido a su política abierta y a sus veinticuatro acuerdos comerciales suscritos a nivel mundial (DIRECON, 2015). El 57\% de las exportaciones chilenas provienen del sector minero, mientras que el sector agropecuario e industrial participa con un $36 \%$ y $7 \%$ respectivamente. Por su parte, Colombia posee diez acuerdos comerciales firmados, entre ellos con la Unión Europea y Estados Unidos; su grado de apertura comercial no supera el 32\% (Delgado y Pérez, 2018); sus exportaciones se componen de café, carbón, petróleo, banano, flores, productos de minería, textiles, cuero y productos químicos. En relación al país vecino, Perú, está inserto en el comercio internacional con la exportación de minerales como cobre, oro, petróleo, cobre refinado y zinc; además, exporta productos agrícolas como café, uvas, frutas y verduras, en que China y Estados Unidos acaparan el $42 \%$ de sus exportaciones; cabe resaltar que entre todos sus productos, el mineral de cobre es el producto estrella de esta economía con una participación del 24,1\% en las exportaciones totales.

No obstante, entre los años 2006 y 2016, a través de un análisis histórico, se puede afirmar que la región suramericana no ha generado cambios estructurales significativos con respecto a su esquema de producción de bienes de bajo valor agregado (Delgado \& Pérez, 2018). 
Tabla 1. Índice PRODY suramericano (2006 y 2016)

\begin{tabular}{llll}
\hline $\begin{array}{l}\text { Descripción del capítulo } \\
\text { arancelario }\end{array}$ & $\begin{array}{l}\text { PRODY 2006 } \\
\text { (miles USD) }\end{array}$ & $\begin{array}{l}\text { PRODY 2016 } \\
\text { (miles USD) }\end{array}$ & Descripción del capítulo arancelario \\
\hline $\begin{array}{l}\text { (1) Aparatos de relojería y sus } \\
\text { partes (Cap. 91) }\end{array}$ & 16,17 & 21,41 & $\begin{array}{l}\text { (1) Residuos y desperdicios de las } \\
\text { industrias alimentarias; alimentos } \\
\text { preparados para animales (Cap. 23) }\end{array}$ \\
\hline $\begin{array}{l}\text { (2) Barcos y demás artefactos } \\
\text { flotantes (Cap. 89) }\end{array}$ & 16,11 & 20,68 & $\begin{array}{l}\text { (2) Tejidos especiales; superficies } \\
\text { textiles con mechón insertado; } \\
\text { encajes; tapicería; pasamanería; } \\
\text { bordados (Cap. 58) }\end{array}$ \\
\hline $\begin{array}{l}\text { (3) Cereales (Cap. 10) } \\
\text { (4) Corcho y sus manufacturas }\end{array}$ & 15,78 & $\begin{array}{l}\text { (3) Lana y pelo fino u ordinario; } \\
\text { hilados y tejidos de crin (Cap. 51) }\end{array}$ \\
$\begin{array}{l}\text { (Cap. 45) } \\
\text { (5) Pasta de madera o de las }\end{array}$ & 20,17 & $\begin{array}{l}\text { (4) Peletería y confecciones de } \\
\text { peletería; peletería facticia o } \\
\text { artificial (Cap. 43) }\end{array}$ \\
$\begin{array}{l}\text { demás materias fibrosas } \\
\text { celulósicas; papel o cartón para } \\
\text { reciclar (Cap. 47) }\end{array}$ & 15,62 & (5) Seda (Cap. 50) \\
\hline
\end{tabular}

Fuente: Delgado y Pérez (2018).

Tabla 2: Índice EXPY suramericano (2006 y 2016)

\begin{tabular}{llll}
\hline País & $\begin{array}{l}\text { EXPY 2006 } \\
\text { (miles de USD) }\end{array}$ & $\begin{array}{l}\text { EXPY 2016 } \\
\text { (miles de USD) }\end{array}$ & País \\
\hline (1) Chile & 13,72 & 17,19 & (1) Chile \\
\hline (2) Argentina & 13,14 & 16,14 & (2) Argentina \\
\hline (3) Brasil & 12,87 & 15,4 & (3) Brasil \\
\hline (4) Perú & 11,9 & 15,11 & (4) Perú \\
\hline (5) Colombia & 11,17 & 13,54 & (5) Ecuador \\
\hline (6) Ecuador & 10,99 & 13,45 & (6) Colombia \\
\hline
\end{tabular}

Fuente: Delgado y Pérez (2018).la Superintendencia de Bancos (2020)—.

Por ello, los resultados arrojan que los cinco productos con el índice PRODY más elevado en el año 2006 fueron aparatos de relojería y sus partes; barcos y demás artefactos flotantes; cereales; corcho y sus manufacturas; así como pasta de madera, demás materias fibrosas celulósicas, papel o cartón para reciclar (ver Tabla 1). Al finalizar el año 2016, este índice es reconfigurado sin salirse de una especialización primaria exportadora tal como se observa en la misma tabla mencionada.

De esta manera, para el año 2016, tal como se observa en la tabla 1, el primer lugar ahora es ocupado por residuos y desperdicios de las industrias alimentarias; le siguió tejidos especiales; lana y pelo fino u ordinario, hilado y tejido de crin; peletería y confecciones de peletería; y también seda. El producto con el menor índice PRODY fueron juguetes, juegos y artículos para recreo o deporte.

Por otro lado, en la tabla (ver Tabla 2) se muestran los índices ExPy calculados, indicador que señala el nivel de ingreso promedio que tiene cada país, el cual exporta una canasta de 
bienes igual o similar al conjunto que se está analizando. Se cuenta que Chile posee el índice EXPY más elevado tanto para el año 2006 como 2016. A la vez, Argentina obtuvo el segundo lugar en estas dos épocas. Para el 2006, Ecuador alcanzó el índice más bajo, mientras que en el 2016 se ubicó en el penúltimo puesto superando a Colombia. En relación a lo expuesto en los resultados del índice PRODY, al ser el cobre uno de los productos con mayor productividad asociada al PIB per cápita, a través del EXPY, Chile demuestra que es el país poseedor de la estructura productiva y especialización comercial más sobresaliente de la región suramericana, en comparación al resto de países. Perú, por su parte, en el año 2006 alcanzó un EXPY de 11,90 miles de dólares y se ubicó detrás de Brasil; a su vez, en 2016 esta brecha disminuyó a o,29 miles de dólares, circunstancia que manifiesta una mejora en el nivel de sofisticación de las exportaciones peruanas.

\section{ESPECIALIZACIÓN PRODUCTIVA NACIONAL}

Sobre los resultados analizados en la sección anterior se puede concluir que aquellos países que se especialicen en la producción de bienes, con PRODY altos, alcanzarán un EXPY más elevado; a la vez, los países que posean un EXPY más elevado, por productividad y eficiencia, que aquel promedio esperado según su PIB per cápita, tendrán mayores tasas de crecimiento económico regional. Esto se evidencia de manera conjunta entre las figuras (ver Figuras 1 y 2), tomadas de Delgado y Pérez (2018), en las que se muestran la relación entre el Ln (EXPY) y el Ln (PIB per cápita) de los años 2006 y 2016 respectivamente. Para el caso específico de Perú, se puede ver que en el año 2006 obtuvo un EXPY de USD 11,90 miles, muy por encima de la tendencia; lo que generó que su especialización productiva de aquel momento promueva un crecimiento acelerado de su PIB per cápita para los próximos años. De esta manera, Perú manifestó un mejor posicionamiento que Ecuador, tal como lo define la teoría; de hecho, si se mantiene este comportamiento peruano, la misma teoría expresa que pronto superará las tasas de crecimiento de Colombia e incluso de Brasil.

Con respecto a Ecuador, el país se ha desenvuelto en un patrón comercial ligado al sector primario, con una estructura productiva de bajo valor agregado, y dentro del cual su producción está sujeta a la exportación de petróleo. Adicionalmente, en el año 2015, el país exportaba setenta y siete productos con ventaja revelada ${ }^{5}$ (OCE, 2016). El índice EXPY para los años 2006 y 2016 ubica a Ecuador en los últimos puestos; según la tabla 2 alcanzó valores de USD 10,99 miles y USD 13,54 miles para el 2006 y 2016 respectivamente. Es así que, a medida que la realidad productiva regional se desarrolla, Ecuador quedaría rezagado dentro de esta carrera. Para el año 2016, solo quince partidas arancelarias aglomeraban las exportaciones nacionales, de las cuales, cinco únicamente estaban por encima de la media regional (Prody). Por consiguiente, en una primera instancia, Ecuador deberá potenciar su producción con mayor nivel tecnológico y con mayor productividad, especialmente en los bienes referentes a las partidas 15, 16, 18, 23 y 71, los cuales, groso modo, se corresponden a productos referentes como cacao, camarones, aceites animales y vegetales, minerales y desperdicios alimenticios.

Por último, para puntualizar esta condición, cabe explicar que las exportaciones ecuatorianas se las caracteriza en dos grandes grupos, por un lado están las exportaciones tradicionales, 
Figura 1. EXPY y renta per cápita de la región suramericana (2006)

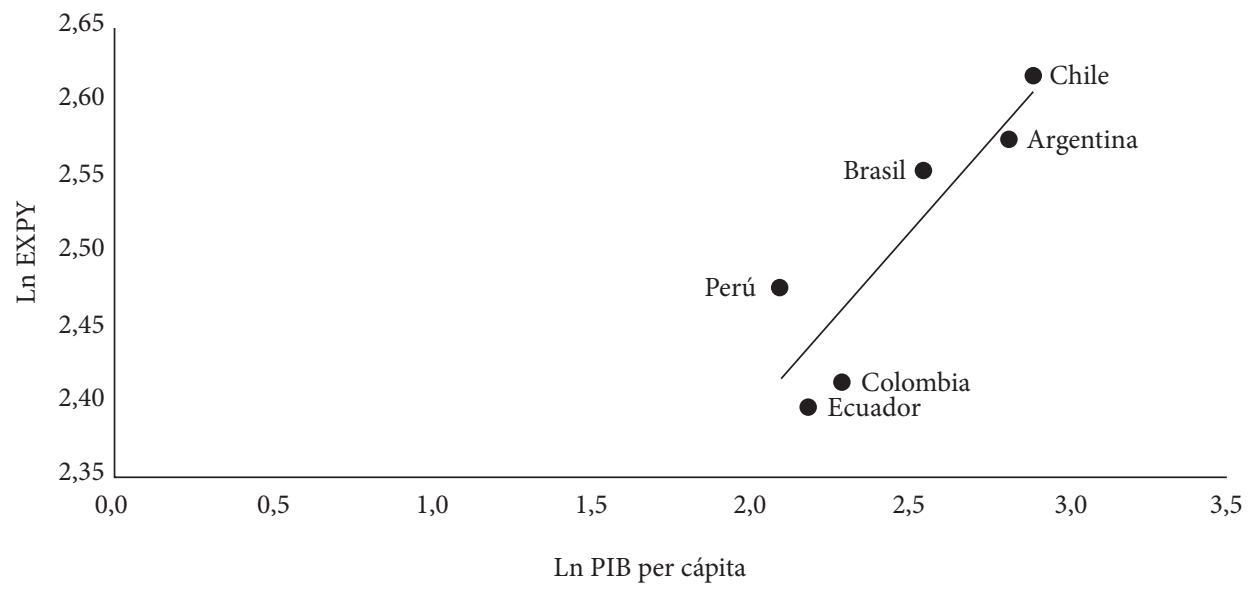

Fuente: Delgado y Pérez (2018).

Figura 2. EXPY y renta per cápita de la región suramericana (2016)

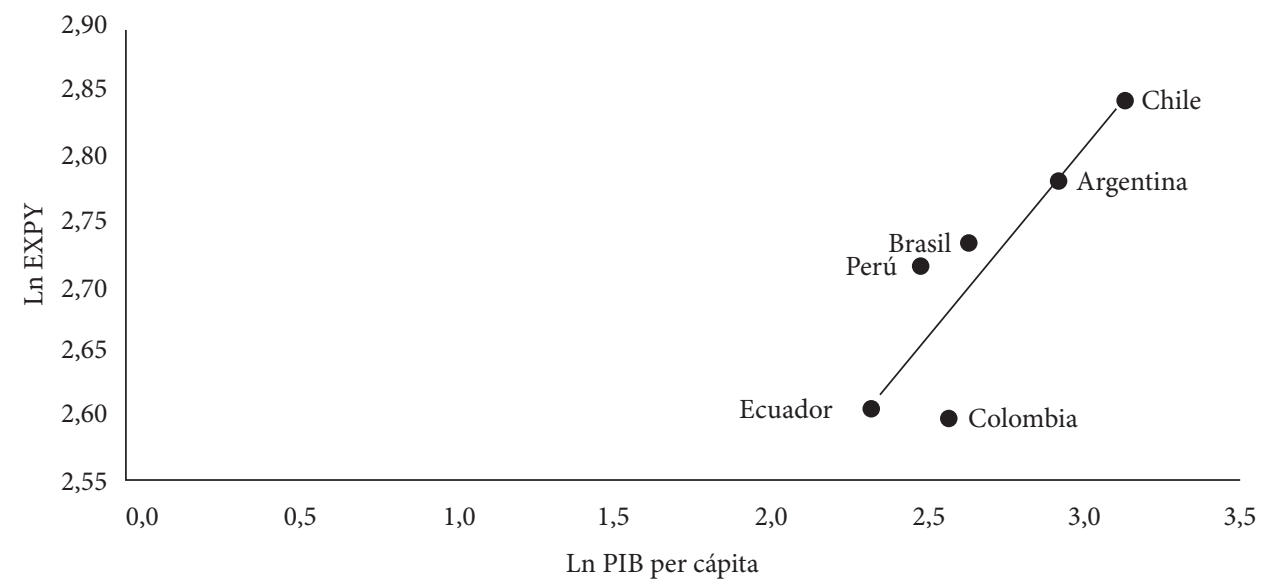

Fuente: Delgado y Pérez (2018).

y por otro están las exportaciones no tradicionales. Éstas últimas, se componen de siete productos primarios y quince que incorporan componente industrial; según estadísticas del Banco Central del Ecuador (BCE, en línea), éstos veintidós productos representan un 24\% de las exportaciones totales. Con respecto a los productos tradicionales, según datos del 2016, los que despuntaron en términos monetarios, fueron el camarón y el banano con USD 2580 y USD 2734 millones respectivamente, de los cuales solo el camarón tiene una importante participación 
dentro de la especialización productiva regional. A éstos, le siguieron productos como cacao, café y atún, los cuales se exportaron en aproximadamente USD 750 millones (вСE, en línea). A partir de ello, debe tenerse presente una vez más el argumento de Hausmann et al. (2005), que exponen que los países que se especializan en productos con alta productividad en relación a su renta per cápita tendrán un mayor impulso en el crecimiento. Con lo cual, Ecuador debe potenciar aún más sus exportaciones tradicionales con la incorporación de mayor componente industrial; posteriormente, luego de este primer momento, como segunda instancia, el país deberá transitar hacia la producción de manufacturas de bajo valor agregado y minería (Delgado y Pérez, 2018).

\section{REFLEXIONES FINALES}

En los últimos diez años la especialización productiva de Suramérica se direccionó hacia la elaboración de bienes con bajo valor agregado. Para el año 2016, los principales productos destinados a la exportación fueron manufacturas que provienen del sector agrícola y minero como cereales, alimentos para animales, tejidos, seda, cobre, estaño y zinc. De manera adicional, se evidenció una reducción del índice PRODY (especialización regional) en productos que en el 2006 tenían mayores niveles de valor agregado como «aparatos de relojería y sus partes» y «barcos y demás artefactos flotantes». Estos productos pasaron de un índice de USD 16,17 miles y USD 16,11 miles a USD 14,58 miles y USD 13,87 miles respectivamente en el 2016.

En cuanto al índice EXPY «especialización nacional a partir de los rubros regionales», Chile, quien alcanzó los mejores valores, pasó de tener un EXPY de USD 13,72 miles a USD 17,19 miles y se mantuvo en primer lugar con dicho crecimiento acumulado del 25\%. Por otro lado, Perú se ubicó en 2006 por encima de Ecuador con USD 0,91 miles y logró en diez años un crecimiento de su EXPY del $27 \%$, incluso superando al crecimiento chileno. De esta manera, la economía peruana evidenció el mejor crecimiento del índice EXPY de la región.

Con respecto a Ecuador, los índices PRODY y EXPY evidenciaron un bajo nivel de sofisticación de su oferta exportable, y, en consecuencia, un limitado crecimiento regional. Con ello, bajo el supuesto de Hausmann et al. (2005), es preciso que Ecuador direccione sus recursos productivos a la producción de bienes que generen mayor renta per cápita, los cuales eventualmente son sugeridos por los resultados del análisis del índice PRODY. Estos bienes se corresponden a partidas arancelarias vinculadas a productos como preparaciones de carne y productos del mar, cacao, grasas y aceites vegetales y animales, productos del sector minero y alimentos preparados para animales. Dentro del marco de la matriz productiva ecuatoriana, en todo caso, es posible sugerir una transición de la estructura productiva agrícola a una especializada que incorpore valor agregado, y también alcance al sector minero. Por lo cual, se sugiere que dentro de una primera fase, el país se vuelque a la exportación de productos tradicionales con mayor componente industrial, y posteriormente tanto a manufacturas de bajo valor agregado como a la producción minera en su segunda fase. 
1 Para una mayor clarificación del índice PRODY, según su desarrollo para j países y para un total de $\mathrm{k}$ productos, se sugiere consultar la sección 3.1 de Delgado y Pérez (2018).

2 Con respecto al índice EXPY, de manera análoga, se aconseja revisar la sección 3.2 de Delgado y Pérez (2018), en el cual se detalla el desarrollo de dicho indicador para un país específico con k productos.

3 La tabla 1, en que se muestra el índice PRODY entre los años 2006 y 2016, fue tomada parcialmente de Delgado y Pérez (2018). En dicho trabajo, se puede observar el índice PRODY de los 97 capítulos arancelarios.

4 La tabla 2, en que se muestra el índice EXPY entre los años 2006 y 2016, fue tomada parcialmente de Delgado y Pérez (2018). Sobre este indicador, como ya se expuso, muestra la incidencia que tienen las exportaciones de cada país dentro de sus respectivos ingresos promedio (PIB per cápita). Chile, al poseer los mayores niveles, indica que posee los productos con mayor especialización regional; con lo cual, su especialización le ha promovido un crecimiento superior al resto de países suramericanos en ambas épocas.

5 De acuerdo al Observatorio de Complejidad Económica (OEC, 2016), la ventaja comparativa revelada es el cociente entre la participación de un producto en las exportaciones de un país y la participación de ese mismo producto en las exportaciones mundiales. Por lo tanto, existe ventaja comparativa revelada en un producto, si es que en relación al total exportado, exporta más que el mundo.

\section{REFERENCIAS}

Banco Central de la República de Argentina. (en línea). BCRA. Recuperado de http://www.bcra.gov.ar/ Banco Central del Ecuador (вСЕ). (en línea). Banco Central del Ecuador. (s. d.).

Delgado, J. y Pérez, J. (2018). Especialización productiva ecuatoriana dentro de la región para una diversificación exportable; (Trabajo de grado en vías de publicación). Recueperada de repositorio digital de la Universidad Central de Ecuador http://www.dspace.uce.edu.ec/

Dirección General de Relaciones Económicas Internacionales (DIRECON). (2015). Informe Anual Comercio Exterior de Chile. Santiago de Chile, Chile: Gobierno de Chile.

Flores, M. y Vaillant, M. (junio de 2011). Cadenas globales de valor y sofisticación de la canasta de exportación en América Latina. Decon. Recuperado de http://cienciassociales.edu.uy/wp-content/ uploads/sites/2/2015/o3/o811.pdf

Hausmann, R., Hwang, J. and Rodrik, D. (December, 2005). What you export matters. Nber working paper series. Recuperado de http://www.nber.org/papers/w11905.pdf

Mayorga, J. Z. y Clemencia, M. (2008). Paul Krugman y el nuevo Comercio Internacional. Criterio Libre, (8), 73-86.

Observatorio de Complejidad Económica (OCE). (2016). Observatorio de complejidad económica. Recuperado de http://atlas.media.mit.edu/es/profile/country/ecu/

Prebisch, R. (1949). El desarrollo económico de la América Latina y algunos de sus principales problemas. CEPAL, (64). Recuperado de http://repositorio.cepal.org/bitstream/handle/11362/40010/1/ prebisch_desarrollo_problemas.pdf 